\title{
HAWK-I: the high-acuity wide-field $K$-band imager for the ESO Very Large Telescope
}

\author{
M. Kissler-Patig ${ }^{1}$, J.-F. Pirard ${ }^{1}$, M. Casali ${ }^{1}$, A. Moorwood ${ }^{1}$, N. Ageorges ${ }^{2}$, C. Alves De Oliveira ${ }^{1}$, P. Baksai ${ }^{2}$, \\ L. R. Bedin ${ }^{1,3}$, E. Bendek ${ }^{2}$, P. Biereichel ${ }^{1}$, B. Delabre ${ }^{1}$, R. Dorn ${ }^{1}$, R. Esteves ${ }^{1}$, G. Finger ${ }^{1}$, D. Gojak ${ }^{1}$, G. Huster ${ }^{1}$, \\ Y. Jung ${ }^{1}$, M. Kiekebush ${ }^{1}$, B. Klein ${ }^{1}$, F. Koch ${ }^{1}$, J.-L. Lizon ${ }^{1}$, L. Mehrgan ${ }^{1}$, M. Petr-Gotzens ${ }^{1}$, J. Pritchard ${ }^{1}$, \\ F. Selman ${ }^{2}$, and J. Stegmeier ${ }^{1}$ \\ ${ }^{1}$ European Organisation for Astronomical Research in the Southern Hemisphere, Karl-Schwarzschild-Str.2, 85748 Garching, \\ Germany \\ e-mail: mkissler@eso.org \\ ${ }^{2}$ European Organisation for Astronomical Research in the Southern Hemisphere, Alonso de Córdova 3107, Vitacura - Santiago, \\ Chile \\ 3 Space Telescope Science Institute, 3700 San Martin Drive, Baltimore, MD 21218, USA
}

Received 4 April 2008 / Accepted 23 September 2008

\section{ABSTRACT}

\begin{abstract}
We describe the design, development, and performance of HAWK-I, the new High-Acuity Wide-field $K$-band Imager for ESO's Very Large Telescope, which is equipped with a mosaic of four $2 \mathrm{k} \times 2 \mathrm{k}$ arrays and operates from $0.9-2.4 \mu \mathrm{m}$ over $7.5^{\prime} \times 7.5^{\prime}$ with $0.1^{\prime \prime}$ pixels. A novel feature is the use of all reflective optics that, together with filters of excellent throughput and detectors of high quantum efficiency, has yielded an extremely high throughput. Commissioning and science verification observations have already delivered a variety of excellent and deep images that demonstrate its high scientific potential for addressing important astrophysical questions of current interest.
\end{abstract}

Key words. instrumentation: miscellaneous - infrared: general

\section{Introduction}

\subsection{HAWK-I in context}

As at the conception of HAWK-I in 2003, the near-infrared imaging capabilities at ESO are provided by SOFI at the New Technology Telescope $\left(1-2.5 \mu \mathrm{m}\right.$; max. field $5^{\prime} \times 5^{\prime}$ with $0.29^{\prime \prime}$ pixels) and ISAAC (1-5 $\mu \mathrm{m}$; max field for $1-2.5 \mu \mathrm{m}$ of $2.5^{\prime} \times 2.5^{\prime}$ with $0.147^{\prime \prime}$ pixels $)$ and NACO $(1-5 \mu \mathrm{m}$; max field $\sim 1^{\prime} \times 1^{\prime}$ but fed by adaptive optics and more typically $20^{\prime \prime} \times 20^{\prime \prime}$ with $\sim 20$ mas pixels) at the Very Large Telescope (VLT).

On average about $15 \%$ of all proposals to ESO request nearinfrared direct imaging with SOFI and ISAAC. Both of these cameras are currently equipped with older $1 \mathrm{k} \times 1 \mathrm{k}$ array detectors, have been in operation for several years (since 1998 and 1999 respectively) and cannot be sensibly upgraded to accommodate the new generation of $2 \mathrm{k} \times 2 \mathrm{k}$ arrays now being installed singly and as mosaics (in WFCAM at UKIRT, NEWFIRM at the KPNO $4 \mathrm{~m}$, WIRCAM at CFHT, VISTA, ..., etc.). All of the mosaic cameras, however, are planned primarily as survey instruments with pixels of $\sim 0.3^{\prime \prime}$ selected for maximum field-of-view and are being installed on $4 \mathrm{~m}$-class telescopes.

From a scientific viewpoint, ISAAC at the VLT has demonstrated the discovery and study potential in many areas of nearinfrared imaging with an $8 \mathrm{~m}$ telescope with the excellent image/seeing quality of the VLT. It has been shown e.g. that deep images requiring a few $\times 10 \mathrm{~h}$ of integration can contribute uniquely to the discovery and study, including morphology, of mature galaxies at $z>3$. Closer to home, it has been used to find and study stellar discs and stellar/planetary mass objects down to $\sim 1$ Jupiter mass. An important general conclusion is that future work in most of these areas will require sampling the best seeing on Paranal and/or require comparable depth but over larger areas than possible with the $2.5^{\prime} \times 2.5^{\prime}$ ISAAC field. In most cases, mosaicing would be prohibitive in integration time or total time (to achieve the required seeing) or would not meet the required photometric or PSF uniformity. There is thus clearly a future scientific requirement for wide field imaging at the VLT. An analysis of all observations performed with SOFI and ISAAC also shows that the $K_{\mathrm{s}}$-band filter has been used most often and about a factor two more than the next most used $J$-band filter. Until the availability of the JWST it is likely that ground based $8 \mathrm{~m}$-class telescopes will provide the best sensitivity achievable in this band (HST is small and warm and SIRTF starts at $\sim 3.5 \mu \mathrm{m}$ ).

From an implementation viewpoint the specifc HAWK-I proposal was triggered by the cancellation of NIRMOS whose four $2 \mathrm{k} \times 2 \mathrm{k}$ buttable near-infrared arrays were already in manufacture at Rockwell (now Teledyne Scientific \& Imaging). With pixels of $\sim 0.1^{\prime \prime}$ sufficient to sample the best Paranal seeing these detectors could provide an (almost) contiguous field of $7.5^{\prime} \times 7.5^{\prime}$ which is about an order of magnitude larger than that of ISAAC. Such a camera is thus an excellent substitute for the lost $J$ - and $H$-band NIRMOS imaging (smaller field but better sampling) and includes the scientifically powerful $K_{\mathrm{s}}$ band. It could also be built at ESO within the financial and manpower resources already committed to build the 4 NIRMOS cameras. 


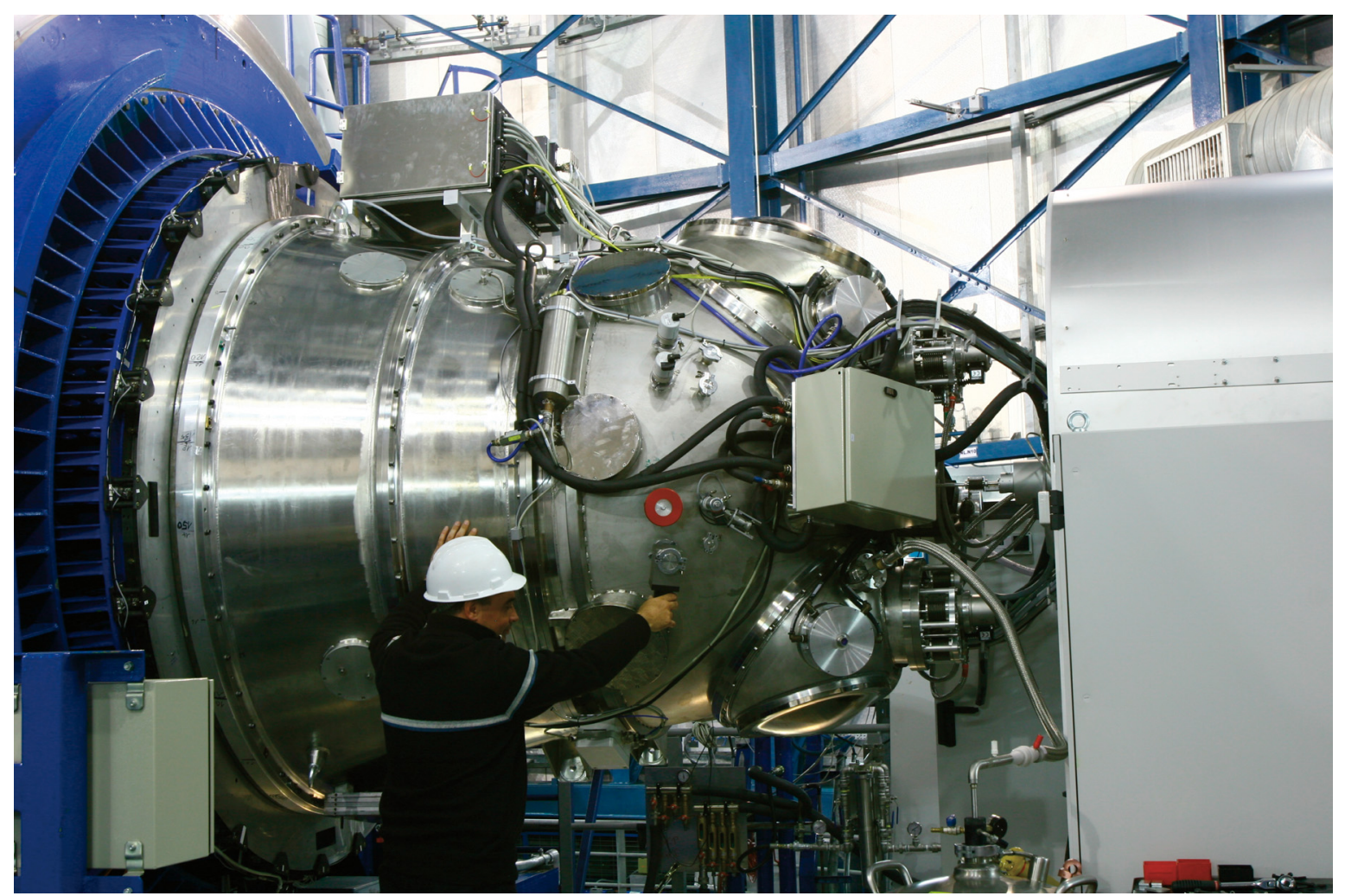

Fig. 1. HAWK-I on the telescope, attached to the Nasmyth focus of the Unit Telescope 4 of the ESO VLT. On the left in blue: the adapter/rotator of the telescope. On the right in light grey: the cable co-rotator fixed on the Nasmyth platform.

Since approval of HAWK-I, the decision has also been taken to convert the telescope on which it is mounted (UT4) to an Adaptive Optics Facility comprising a deformable secondary mirror, four laser guide stars and wavefront sensor units for HAWK-I (and an wide-filed integral field spectrograph: MUSE). This will provide ground layer adaptive optics correction over its full field bringing improved seeing for longer periods and further enhancing its imaging capability and point source sensitivity (see Sect. 4).

In summary therefore, the scientific case for near-infrared imaging at the VLT was strong and the specific HAWK-I camera as proposed is attractive in that it provides a ten times larger field, higher efficiency and better image quality than ISAAC; substitutes the lost NIRMOS $J$-, $H$-band capability and adds the important $K_{\mathrm{S}}$ band; will profit from ground layer adaptive optics in the future and could be built using already allocated resources.

\subsection{The science case}

Science cases for wide-field near-infrared instruments have already been addressed above. Given the wide field, fine sampling and high sensitivity of HAWK-I, the deepest scientific impact is expected in the areas of surveys of faint sources. Primarily deep multi-colour galaxy evolution surveys will profit from the new facility, including morphological studies of mature galaxies in the redshift range 1.5 to 4 . In that respect, HAWK-I will provide the completement to large field optical surveys such as the GOODS Chandra/SIRTF field that is currently inefficiently covered by ISAAC. Another strong science case in the high redshift universe is provided by the cosmological narrow band filters that will allow efficient surveys of emission line objects at redshifts 7 to 9, exploring the very first star forming objects in our universe. But also multi-wavelength observations of nearby normal and active galaxies will be enhanced by HAWK-I, whose field is better matched to the ground-based wide-field optical imagers in order to provide a deep view into the closest resolved stellar populations beyond the Milky Way. Further, HAWK-I will be very well suited for the search in our Galaxy of the most massive (embedded OB) stars and least massive objects (brown dwarfs, planetary mass objects), leading to a better understanding of the stellar IMF. Finally, HAWK-I will also be a perfect instrument for the study of outer solar system bodies, such as distant, icy minor bodies, or the study of long period comets. Clearly, HAWK-I will allow rapid progress in very diverse areas of modern astronomy by filling the niche of a wide-field, well sampled near-infrared imager on an $8 \mathrm{~m}$-class telescope.

\section{HAWK-I instrument description}

\subsection{General overview}

HAWK-I is a cryogenic instrument now installed on the adapter/rotator of one of the Nasmyth foci of the VLT Unit Telescope 4 (UT4) as shown in Fig. 2. An overview of the HAWK-I characteristics is presented in Table 1.

With the exception of the entrance window, the optical design is based on an all reflective configuration. The purpose of the optical configuration is to adapt the $F$-number of the input beam to the pixel field-of-view requirement $\left(0.1^{\prime \prime} /\right.$ pixel $)$ and to limit the stray light reaching the detector by means of a cold field stop located at the entrance of the instrument and the third mirror acting as a cold pupil stop.

Just before the light reaches the detectors, two filter wheels allow the insertion of Broad Band $\left(Y, J, H, K_{\mathrm{s}}\right)$ and Narrow Band (interstellar lines and cosmological) filters. 


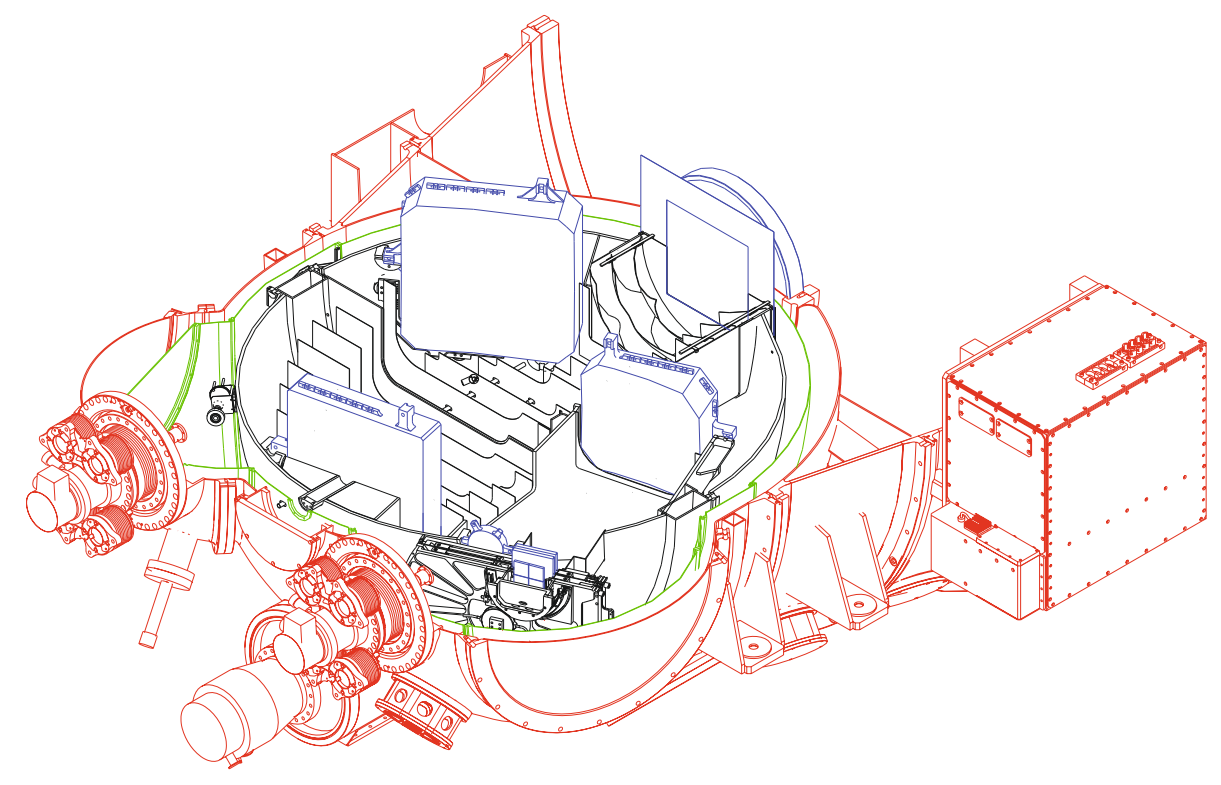

Fig. 2. Cut through HAWK-I for an optical and mechanical overview. Blue: optical components; black: cold assembly, filter wheels, detector assembly; green: radiation shield; red: vessel structure, cryogenic components, electronic rack.

Table 1. HAWK-I properties.

\begin{tabular}{ll}
\hline \hline Parameter & Value \\
\hline Scale & $106 \mathrm{mas} /$ pixel \\
Field of view & $7.5^{\prime} \times 7.5^{\prime}$ \\
Image quality $(80 \% \mathrm{EE})$ & $<0.2^{\prime \prime}$ \\
Distortion & $<0.3 \%$ across the field \\
Optics throughput (w/o detector) & $>70 \%$ \\
Filters & $4 \mathrm{Broad}$ band, 6 Narrow band \\
Detectors & four $2 \mathrm{k} \times 2 \mathrm{k}$ Hawaii2RG arrays \\
Detector quantum efficieny & $>80 \%$ \\
Detector temperature & $75 \mathrm{~K} \pm 1 \mathrm{mK}$ \\
Read noise & $\sim 5 \mathrm{e}^{-}$for integration times $>15 \mathrm{~s}$ \\
Instrument background & $\sim 0.10-0.15 \mathrm{e}^{-} / \mathrm{s}$ \\
Instrument temperature & $<140 \mathrm{~K}$ \\
\hline
\end{tabular}

The HAWK-I focal Plane is equipped with a mosaic of four $2 \mathrm{k} \times 2 \mathrm{k}$ Rockwell HgCdTe Molecular Beam Epitaxy (MBE) HAWAII 2 RG arrays. The packaging of this $2 \times 2$ mosaic detector is provided by GL Scientific re-using the design developed for the JWST program. The acquisition system is based on the IRACE system (Infrared Array Control Electronics) developed at ESO.

The optics and focal plane are mounted on a spherical cold structure which provides the mechanical stability between the different elements. This assembly is cooled to cryogenic temperature using a liquid nitrogen pre-cooling circuit and two closed cycle coolers. The detectors and the filter wheel unit are connected to the second stage of the Closed Cycle Cooler and operated at a temperature close to $75-80 \mathrm{~K}$. A temperature below $140 \mathrm{~K}$ is achieved for the rest of the instrument.

The vacuum vessel containing the instrument is interfaced to the Nasmyth adaptor. The interface flange is designed such that it can accommodate the further installation of wavefront sensors required for the operation with a later adaptive secondary mirror of the telescope.

The specification of the camera was largely driven by the four $2 \mathrm{k} \times 2 \mathrm{k} 0.9-2.5 \mu \mathrm{m}$ Rockwell array detectors, i.e. the facts that $i$ ) four was the largest number of arrays we could mosaic and $\mathrm{ii}$ ) we wished to be seeing limited under the best conditions on Paranal. We thus specified $0.106^{\prime \prime}$ pixels (cf. ISAAC $0.147^{\prime \prime}$ ) which yields a field of $7.5^{\prime} \times 7.5^{\prime}$. Moreover, this pixel sampling of $0.106^{\prime \prime}$ is fully compatible with the expected wide field image quality improvement provided by the Adaptive Secondary mirror (to come around 2012) without any loss of resolution.

HAWK-I is also designed to work in future with a groundlayer adaptive optics module (GRAAL) as part of the Adaptive Optics Facility (Arsenault et al. 2006) for the VLT. The facility is expected at the telescope around 2012, at which point the GRAAL module will be brought into operation. This module is inserted between the telescope adapter/rotator and the HAWK-I entrance window and is equipped to sense the wave-front of four laser guide stars and one natural guide star and to feed back corrections to the adaptive secondary mirror of the VLT. The module aims at correcting the ground-layer turbulence over the complete field-of-view, i.e. not at achieving diffraction limited images but rather at improving significantly the seeing/image quality of the near-infrared images.

\subsection{Optical design}

An important task of the preliminary design phase was to perform a selection between a full dioptric design and a catadioptric design. Both configurations were satisfactory in terms of optical requirements. Finally, the preference was given to the reflective design for the following reasons:

- the reduction of the potential development risks in relation with the poor knowledge of $\mathrm{d} n / \mathrm{d} T$ behavior of infrared material at cryogenic temperature;

- the removal of all potential risks of lens misalignment/breakage during integration transportation, earthquake and thermal transition;

- a higher throughput without the development of any specific anti-reflection coating;

- the confidence, based on previous experience at ESO, that is possible to manufacture aluminum alloy mirrors with a very good optical quality and without significant deformation at cryogenic temperature;

- a large reduction of the potential problems linked to ghosts. 


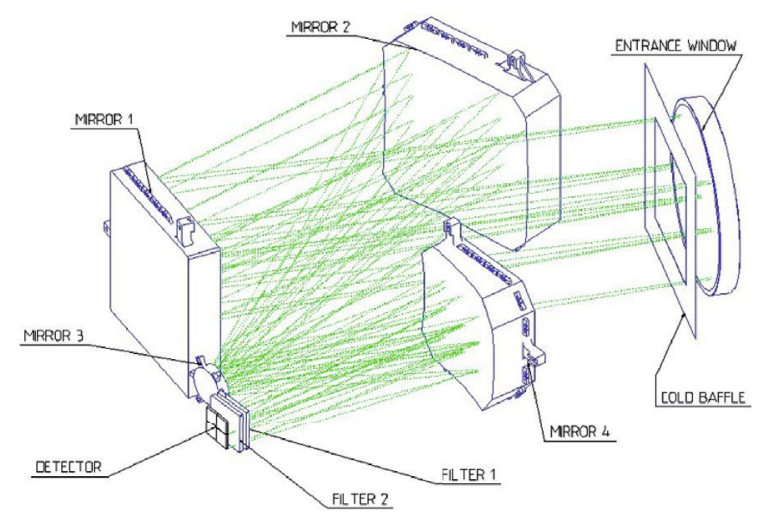

Fig. 3. HAWK-I optical layout.

The catadoptric solution has also some drawbacks:

- the size of the vessel is larger to accommodate the optical layout. This leads to an increase of the total mass of the instrument and consequently to some increase in cost;

- the mirror solution introduces some limited distortion $(0.1 \%)$ that needs to be corrected before stacking jittered images. However, this is easily handled by modern data processing tools.

An overview of the final optical layout is given in Fig. 3. The entrance window of the vacuum vessel is used to image the pupil on the M 3 mirror. A cold baffle stops the light outside of the instrument field of view. The first folding mirror (M1) is used for beam accommodation. Then, the camera consists of one large spherical mirror M 2 and two aspherical mirrors M 3 and M4 allowing to adapt the telescope beam to the required $F / 4.36$.

The mirrors were made by Axsys Technologies by diamond turning a thin layer of nickel supported on machined aluminium alloy blanks which had been first stress relieved by thermal cycling. They were finally gold coated to maximise their infrared reflectivity and are attached to the cold structure by isostatic mounts to avoid any degradation of the optical quality. The two filter wheels are located directly in front of the detector mosaic. The size of the filter needed to cover the whole field of view is $105 \times 105 \mathrm{~mm}$. The beam incidance angle versus the filter is quasi constant for all points of the field to keep the spectral filtering uniform over the whole field of view.

\subsection{Mechanical design}

The HAWK-I instrument is centred on the Nasmyth adaptor by the interface flange. The vacuum vessel is split into three elements, the:

- vessel front part which extends to the Interface flange diameter. This area is reinforced to provide sufficient stiffness for the integrated entrance window and to the centre part connection flange;

- vessel centre part which offers all necessary supply ports and the mechanical connections to support the cold mechanics;

- access to the detector, filter unit, filter exchange, the connection of the closed cycle coolers, pre-cooling lines and electrical cables.

The cold assembly (Fig. 4) is built from two hemispherical shells made from aluminum and supports inside, the Al mirrors and outside the filter wheel unit. The cold structure is made of aluminum and behaves thermally like the mirror material. The interface between the vessel and the cold structure is made via

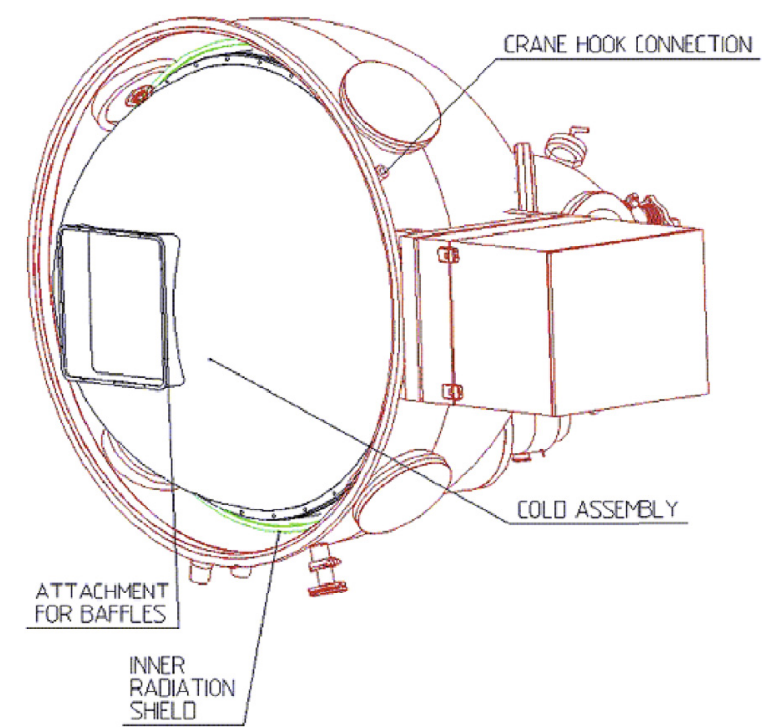

Fig. 4. HAWK-I cold assembly.

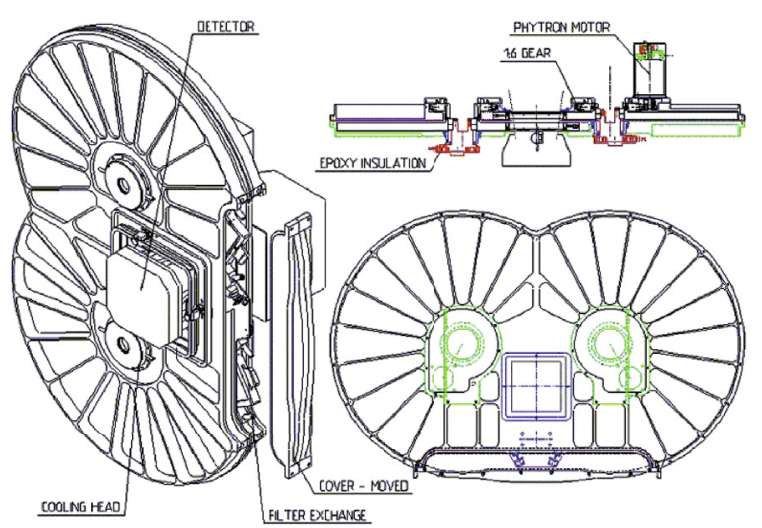

Fig. 5. HAWK-I filter wheel assembly.

5 epoxy spiders which provide a good thermal insulation and sufficient stiffness. A radiation shield inserted between the vessel and the cold structure is connected to the closed cycled cooler to reduce the vessel thermal radiation.

The filter wheel unit houses two filter wheels with six filter slots each - one of the slots being used as an open position in each wheel (Fig. 5). The filter assembly is attached tangentially to the wheel to allow for the easy exchange of filters. The filter wheels are rotated by Phytron stepper motors via a pre-loaded gear system.

The detector mount (Fig. 6) is attached at the back of the filter wheel unit. The detectors are cooled down via the second stage of the close cycle cooler.

\subsection{Cryo-vacuum design}

The cryo-vacuum design is based mostly on the successful aspects of the other ESO cryogenic instrument. In addition to the standard pumping system, an emergency back-up system provides some extra safety for the expensive detector system.

The cooling system is based on two Leybold closed cycle coolers to maintain the instrument below $140 \mathrm{~K}$ and the detector and the filters at about $75 \mathrm{~K}$. The two Leybold heads are fed with a single compressor. 


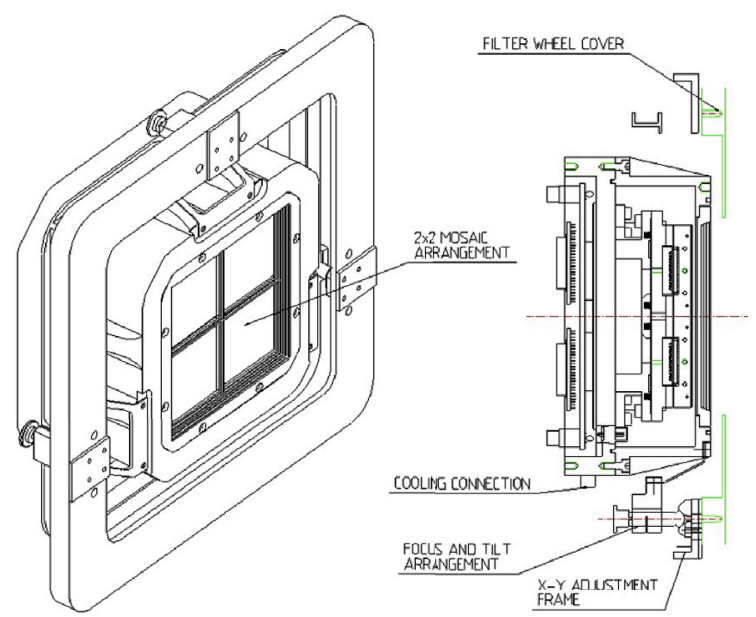

Fig. 6. HAWK-I detector mount.

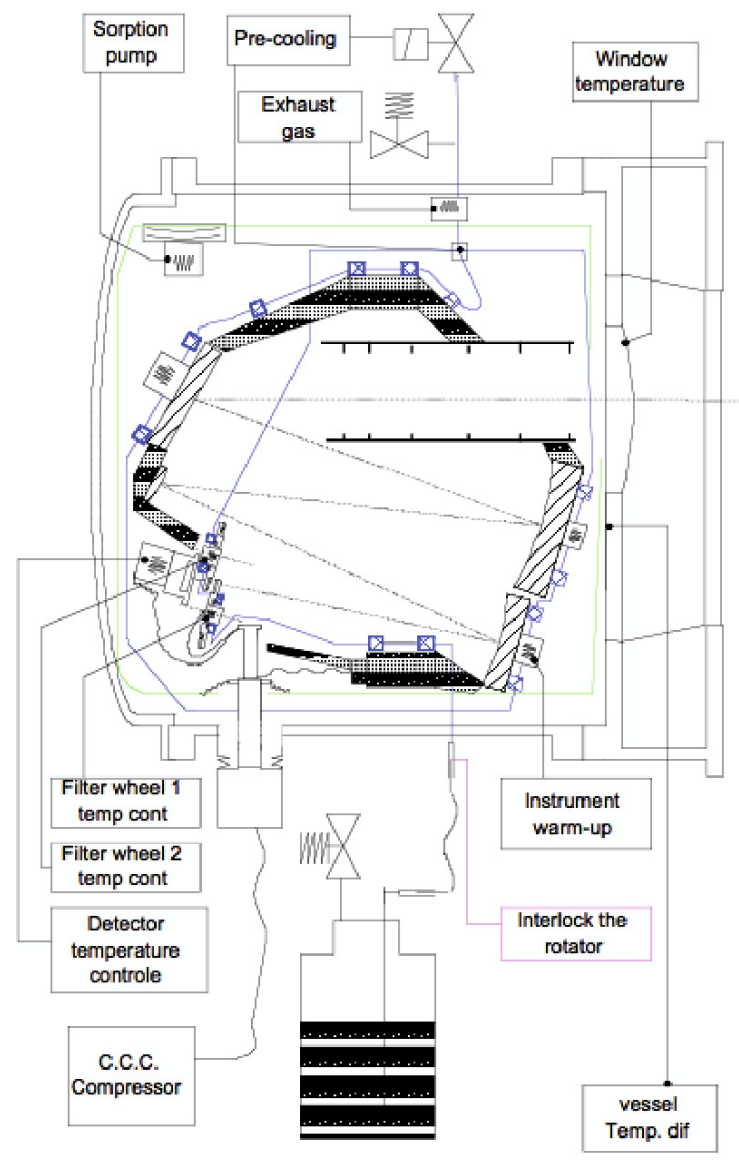

Fig. 7. HAWK-I overview of the cryogenic design.

A liquid nitrogen pre-cooling circuit accelerates cool-down $(<24 \mathrm{~h})$. Because of the large size of the entrance window a defogging system is activated when the temperature difference between the window and the dew point is too small. Figure 7 shows an overview of the cryogenic design.

\subsection{Detector and acquisition design}

The HAWK-I detector mosaic is composed of four $2 \mathrm{k} \times 2 \mathrm{k}$ Rockwell $\mathrm{HgCdTe} \mathrm{MBE}$ buttable arrays with $2.5 \mu \mathrm{m}$ cutoff. GL scientific in Hawaii provided the packaging into a

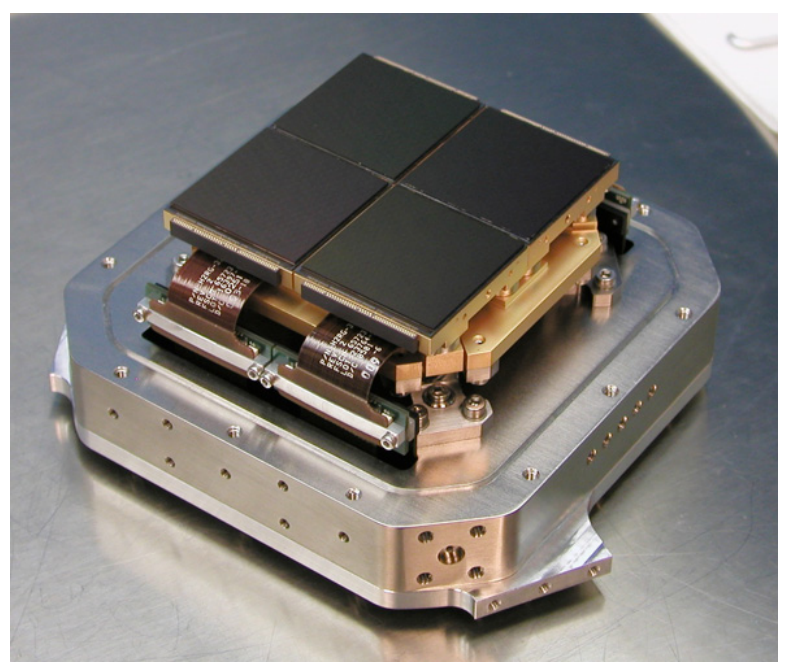

Fig. 8. HAWK-I detector mount.

$2 \times 2$ mosaic using a design developed for the JWST (Fig. 8). The fan-out board was developed for our application at ESO.

The acquisition system is able to acquire 132 channels simultaneously. This allows to read out the full detector in $1.3 \mathrm{~s}$ which is adequate for all HAWK-I applications. For the foreseen ground-layer adaptive optics system expected with the advent of the adaptive secondary mirror in 2012, a fast read out of subwindow (detector guiding mode) is available to acquire a Natural Guide Star and perform the tip-tilt correction at a frequency of about $100 \mathrm{~Hz}$.

\section{HAWK-I performance}

This section summarises the main characteristics of HAWK-I and its performance as measured during commissioning. More details and updates are available through the instrument web pages at ESO (wwW . eso.org/instruments/hawki).

\subsection{Detector characteristics and field-of-view}

\subsubsection{Field-of-view coverage}

The $7.5^{\prime} \times 7.5^{\prime}$ Field of View of HAWK-I is covered by four Hawaii-2RG chips of $2048^{2}$ pixels each (1 pixel corresponds to $0.106^{\prime \prime}$ on the sky). The detectors are separated by gaps of about $15^{\prime \prime}$ as shown in Fig. 9. The figure also shows the naming convention of the four detectors. The images of the four detectors are stored together in a single FITS file as four extensions. Note that quadrants 1, 2, 3, 4 are usually, but not necessarily, stored in extensions 1, 2, 4, 3 of the HAWK-I FITS file ${ }^{1}$.

Finally, due to necessary baffling in the all-reflective optical design of HAWK-I, some vignetting at the edges of the field has turned out to be inevitable due to positioning tolerances of the light baffles. The vignetting measured on sky is summarised in Table 2. Note that although the $+\mathrm{Y}$ edge vignetting is small in amplitude, it extends to around 40 pixels at $<10 \%$.

\footnotetext{
${ }^{1}$ Re-naming the quadrants would have caused confusion in the design documentation; re-ordering the quadrants in the detector electronics would generated read-out overhead; and last but not least, the FITS convention forbids to identify extensions by their location in the file. For unambiguous identification, the FITS keyword EXTNAME in each extension should be used (e.g. EXTNAME = CHIP1.INT1, referring to Q1 or chip\#66).
} 

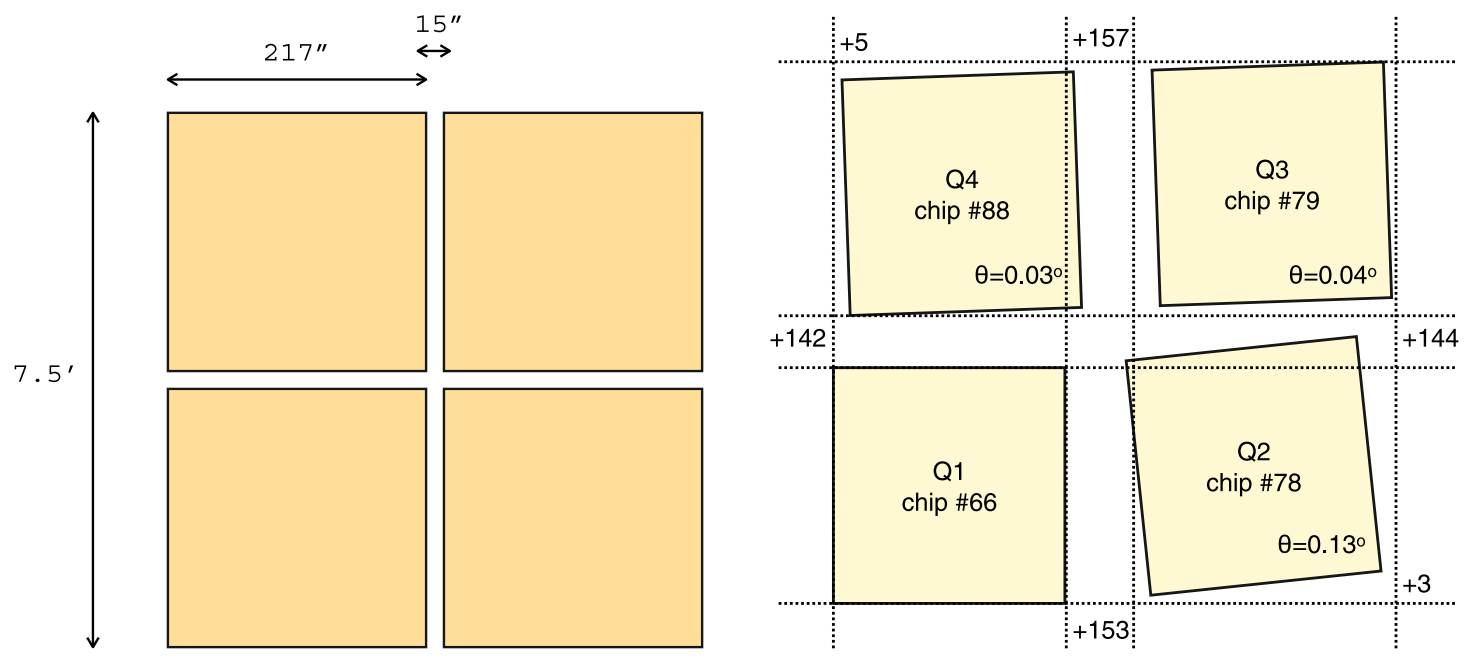

Fig. 9. HAWK-I field-of-view coverage by the detector mosaic. Left: the layout of the field of view on the sky - note the small gap of $\sim 15^{\prime \prime}$ between the four detectors. Right: the relative orientation of the four detectors with the average gap size given in pixels.

Table 2. HAWK-I field-of-view vignetting.

\begin{tabular}{ccc}
\hline \hline Edge & $\begin{array}{c}\text { No of columns } \\
\text { or rows vignetted }>10 \%\end{array}$ & $\begin{array}{c}\text { Maximum } \\
\text { vignetting }^{a}\end{array}$ \\
\hline$+\mathrm{Y}$ & 1 & $14 \%$ \\
$-\mathrm{Y}$ & 8 & $54 \%$ \\
$-\mathrm{X}$ & 7 & $36 \%$ \\
$+\mathrm{X}$ & 2 & $15 \%$ \\
\hline
\end{tabular}

a The last column represents the maximum extinction of a vignetted pixel, i.e. the percentage of light absorbed in the pixel row or column, with respect to the mean of the field.

Table 3. HAWK-I detector characteristics.

\begin{tabular}{|c|c|c|c|c|}
\hline Detector parameter & $Q 1$ & $Q 2$ & $Q 3$ & $Q 4$ \\
\hline Detector numbering & \#66 & $\# 78$ & \#79 & $\# 88$ \\
\hline Operating temperature & \multicolumn{4}{|c|}{$75 \mathrm{~K}$, controlled to $1 \mathrm{mK}$} \\
\hline Gain [e $\left.\mathrm{e}^{-} / \mathrm{ADU}\right]$ & 1.705 & 1.870 & 1.735 & 2.110 \\
\hline Dark current (at $75 \mathrm{~K}$ ) & \multicolumn{4}{|c|}{$<0.01 \mathrm{e}^{-} / \mathrm{s} / \mathrm{pix}$} \\
\hline Read-out noise (NDR) ${ }^{a}$ & \multicolumn{4}{|c|}{$\sim 5$ to $12 \mathrm{e}^{-}$} \\
\hline Linear range (1\%) & \multirow{3}{*}{\multicolumn{4}{|c|}{$\begin{array}{l}\quad 60000 \mathrm{e}^{-}(\sim 30000 \text { ADUs }) \\
\text { between } 40000 \text { and } 50000 \text { ADUs }\end{array}$}} \\
\hline Saturation level & & & & \\
\hline Hot/dead pixels & & & & \\
\hline
\end{tabular}

${ }^{a}$ The read-ou noise (RON) in the default Non-Destructive Read (NDR) mode depends on the DIT: the detector is read continuously every $\sim 1.3 \mathrm{~s}$, i.e. the longer the DIT, the more reads are possible and the lower the RON. For the minimum DIT ( $1.3 \mathrm{~s})$, the RON is $\sim 12 \mathrm{e}^{-}$; for DIT $=10 \mathrm{~s}$, the RON is $\sim 8 \mathrm{e}^{-}$and for DIT $>15 \mathrm{~s}$, the RON remains stable at $\sim 5 \mathrm{e}^{-}$.

\subsubsection{Detector characteristics}

The characteristics of the four detectors are summarised in Table 3. Note that for detector integration times (DIT) greater than $15 \mathrm{~s}$ the read-out noise (RON) of the detector is as low as $\sim 5 \mathrm{e}^{-}$. The Hawaii2RG detectors have four reference columns/rows around each device which are not sensitive to light.

\subsubsection{Threshold limited integration}

The HAWK-I detector is operated by default in a non-destructive read-out mode (see above). For HAWK-I, we have implemented a variation of this mode: the Threshold Limited Integration

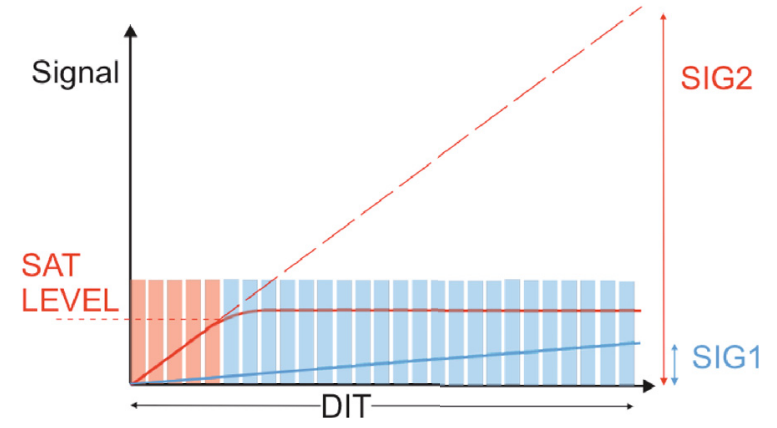

Fig. 10. HAWK-I's Threshold Limited Integration mode. Only pixel values below SATLEVEL are taken into account for calculation of slope for pixels with high flux (red line). For low flux pixels (blue line) all non-destructive readouts indicated by rectangles are used. The final FITS file contains the values SIG1 and SIG2, allowing to exceed the saturation level of the detector.

(TLI), (see Fig. 10 and Finger et al. 2008, for a detailed description).

For a non-destructive read-out, it is possible to define a threshold (e.g. a value below the saturation limit) above which the read-out values are not taken into account anymore. I.e. for a bright star reaching the saturation limit during the detector integration time (DIT), only the values read before the star goes into saturation are taken into account (see red line in Fig. 10), and the pixel value written into the FITS file is the value extrapolated to the full integration time (calculated from the slope of the non-saturated, non-destructive read-outs). All pixels which have absolute ADU values below this threshold over the DIT are processed normally (blue pixel in Fig. 10).

The great advantage is of course that most bright objects do not saturate in the final FITS file and that (depending on the DIT) the dynamic range is increased (by typically 2 mag for a 30 s DIT). The pixels may be physically saturated of course, i.e. the persistence (which is important for highly saturated pixels in HAWK-I) remains. Another caveat is that the effective integration time for bright stars is not the DIT, but the time until the star reached the saturation limit - which will alter the noise properties for bright stars - although not critically as the $S / N$ is high. Finally, a very bright star (of the order of $<9$ mag) will 
Table 4. HAWK-I filter summary.

\begin{tabular}{ccccccc}
\hline \hline Filter name & $\begin{array}{c}\text { Central } \\
\text { wavelength }[\mathrm{nm}]\end{array}$ & $\begin{array}{c}\text { Cut-on } \\
(50 \%)[\mathrm{nm}]\end{array}$ & $\begin{array}{c}\text { Cut-off } \\
(50 \%)[\mathrm{nm}]\end{array}$ & $\begin{array}{c}\text { Width } \\
{[\mathrm{nm}]}\end{array}$ & $\begin{array}{c}\text { Tansmission } \\
{[\%]}\end{array}$ & Comments \\
\hline$Y$ & 1021 & 970 & 1071 & 101 & $92 \%$ & $\begin{array}{c}\text { out-of-band transmission of } 2 \times 10^{-4} \\
\text { (or 0.04\%) between } 2.3 \text { and } 2.7 \mu \mathrm{m}\end{array}$ \\
$J$ & 1258 & 1181 & 1335 & 154 & $88 \%$ & \\
$H$ & 1620 & 1476 & 1765 & 289 & $95 \%$ & \\
$K_{\mathrm{s}}$ & 2146 & 1984 & 2308 & 324 & $82 \%$ & \\
\hline $\mathrm{CH}_{4}$ & 1575 & 1519 & 1631 & 112 & $90 \%$ & \\
$\mathrm{Br} \gamma$ & 2165 & 2150 & 2181 & 30 & $77 \%$ & \\
$\mathrm{H}_{2}$ & 2124 & 2109 & 2139 & 30 & $80 \%$ & \\
\hline $\mathrm{NB} 1060$ & 1061 & 1057 & 1066 & 9 & $70 \%$ & Ly $\alpha$ at $z \sim 7.7$ \\
$\mathrm{NB} 1190$ & 1186 & 1180 & 1192 & 12 & $75 \%$ & Ly $\alpha$ at $z \sim 8.7$ \\
$\mathrm{NB} 2090$ & 2095 & 2085 & 2105 & 20 & $81 \%$ & H $\alpha$ at $z \sim 2.2$ \\
\hline
\end{tabular}

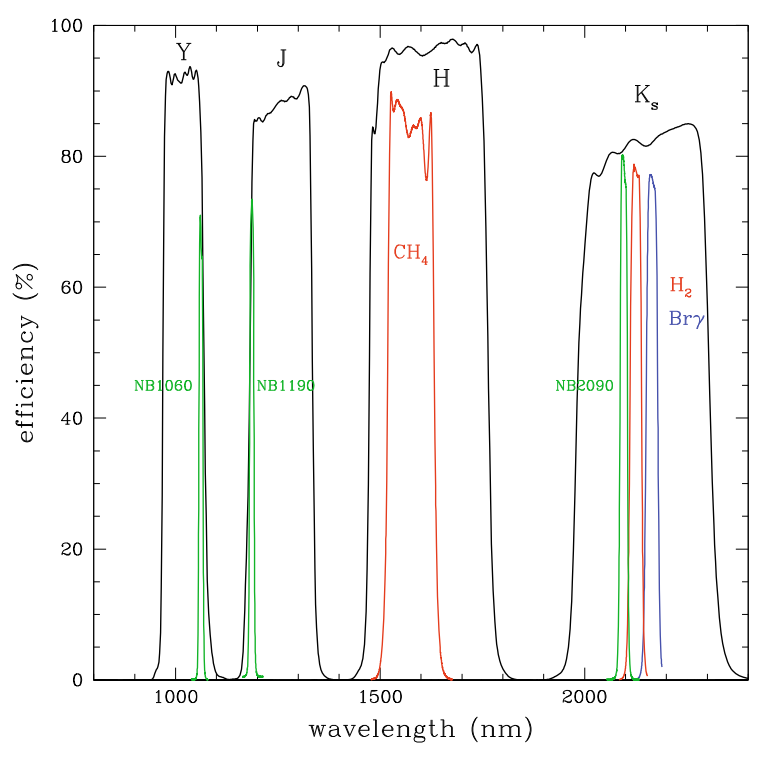

Fig. 11. HAWK-I filter transmission curves.

saturate within the first non-destructive read and will not profit from the TLI mode.

Tests have verified that the photometry in not altered at a detectable level for stars above the threshold. Yet, it is recommended that for precise photometry $(<5 \%)$ only stars below the threshold (typically set to 30000 ADU) be used.

A user that does not want to make use of this mode, can simply flag all pixels above the value recorded in the header keyword DET SATLEVEL (typically 30000 ) as saturated.

\subsection{Filter system}

HAWK-I hosts two filter wheels of six position each. Each wheel has an open position, leaving room for two times five filters: 4 broad-band filters, and 6 narrow-band filters.

The broad-band filters $Y, J, H, K_{\mathrm{s}}$ were ordered following the Mauna Kea Observatory specifications (see Tokugana \& Vacca 2005; Simons \& Tokugana 2002). This standardisation allows an easy cross-calibration and comparison between HAWK-I and the UKIDSS survey (Hewett et al. 2006) as well as the VISTA infrared camera (Dalton et al. 2006).

The narrow-band filters include three cosmological filters for Ly $\alpha$ at $z \sim 7.7$ (at $1.06 \mu \mathrm{m}$ ) and $z \sim 8.7$ (at $1.19 \mu \mathrm{m}$ ), and $\mathrm{H} \alpha$ at $z \sim 2.2$ (at $2.09 \mu \mathrm{m})$ as well as three "galactic" filters $\left(\mathrm{CH} 4, \mathrm{H}_{2}\right.$,
Table 5. HAWK-I limiting magnitudes and saturation limits $(S / N=5$ for $1 \mathrm{~h}$ on source, $0.8^{\prime \prime}$ seeing, 1.2 airmass).

\begin{tabular}{lccc}
\hline \hline Filter & $\begin{array}{c}\text { Limiting mag } \\
\text { [Vega] }\end{array}$ & $\begin{array}{c}\text { Limiting mag } \\
{[\mathrm{AB}]}\end{array}$ & $\begin{array}{c}\text { Saturation limit } \\
\text { (in 2 s) }\end{array}$ \\
\hline$J$ & 23.9 & 24.8 & 11.0 \\
$H$ & 22.5 & 23.9 & 11.3 \\
$K_{\mathrm{s}}$ & 22.3 & 24.2 & 10.2 \\
\hline
\end{tabular}

$\operatorname{Br} \gamma$ ). The filter properties are summarised in Table 4, and the filter transmission curves are shown in Fig. 11. The transmission curves are available in electronic form on the instrument web pages (WWW . eso.org/instruments/hawki/).

The proximity of the detectors and the filters could generate optical ghosts. Ghosts (out of focus images showing the M 2 and telescopespiders) have only been be found in images of bright stars taken with the NB1060 and NB1190 filters: their total integrated intensities are, in both cases, around $2 \%$, while their surface brightness is a factor $10^{-4}$ lower than the peak brightness of the stellar PSF.

\subsection{Photometric performance}

\subsubsection{Limiting magnitude}

HAWK-I is a highly effective imager with a total instrument throughput, including detectors, close to $60 \%$ in the $Y, J$, $H$ bands, and over $50 \%$ in the $K_{\mathrm{s}}$ band.

The limiting magnitudes are of course very much dependent on the observing conditions. The exposure time calculator, available through the ESO web pages (www.eso.org/observing/ etc/), is well calibrated and can be used to estimate the performance in all filters.

As an example, we give in Table 5 the limiting magnitudes ( $S / N=5$ for a point source in $3600 \mathrm{~s}$ integration on source) and saturation limits for broad-band filters in average conditions (0.8" seeing, 1.2 airmass).

\subsubsection{Calibrating photometry with HAWK-I, using the 2MASS catalogue}

A calibration plan assures that the performance is monitored every night and has shown that HAWK-I is extremely stable.

Calibration of the photometry to $\sim 5 \%$ across the field, can be achieved in two ways. Either photometric standard stars from the standard catalogues (UKIRT faint standards, 
Hawarden et al. 2001; Persson list of standards, Persson et al. 1998) can be measured before/between/after the observations, moving the standard stars through all four quadrants.

The numerous 2MASS calibrated stars present in the frame can also be used to derive the zero points. Indeed, the HAWK-I field of view is large enough to usually include 10 to 100 calibrated 2MASS stars. Each star cannot be measured with the precision of a photometric standard star, but is typically catalogued with photometry good to $<0.1 \mathrm{mag}$. The large number across the field permits the determination of the zero point on the image to $\sim 0.05 \mathrm{mag}$, using these local secondary standards. Extinction coefficients are then automatically taken into account and the colour terms for HAWK-I can also be derived.

The above determination of the zero points and colour terms should be performed after the illumination correction. For this purpose, an illumination map is built by observing stars in a grid on each detector. Zero points are derived as a function of position on the chip and a map is interpolated. The variations of the zero point are typically smaller than a few percent in the broad-band filters.

\subsection{Astrometric performance}

For a full analysis of the astrometric performance of HAWK-I, we refer to Anderson et al. (2008). Here we draw attention to a few points to note when translating detector coordinates into relative or absolute coordinates on the sky.

\subsubsection{Centre of rotation and centre of pointing}

The optical axis of the telescope is not perfectly aligned with the one defined by the centre of the detector mosaic. The difference being small, this is not physically corrected for by the telescope control software. In the standard orientation (North is $+\mathrm{Y}$, East is $-\mathrm{X}$ ) the centre of the detector array is located $\sim 0.4^{\prime \prime}$ East and $\sim 0.4^{\prime \prime}$ South of the telescope pointing, i.e. shifted by 4 pixel on each axis.

The world coordinate system (WCS) provided in the header of each quadrant takes this into account, however. Further, the WCS of each quadrant is defined such that it refers (cf. CRPIX keywords) to the reference pixel taken to be the central pointing (and centre of rotation) of the telescope (i.e. the TEL.TARG.RA and TEL.TARG.DEC keywords reported in the header).

\subsubsection{Distortion correction}

The optical distortions across the HAWK-I field of view are very small $(0.1 \%$ at the worst $)$. They were measured following the auto-calibration method of Anderson et al. (2006) and we refer to Anderson et al. (2008) for the full details. The worst variation of the non-linear part of the distortions from the centre of the detector is $\sim 2.9$ pixels, for a diagonal of a quadrant of $\sim 2900$ pixels.

A distortion map (shown in Fig. 12) is produce regularly in the form of a look-up table of corrections to be applied to the $x$ and $y$ coordinates (as a function of position in the field) in order to recover the undistorted position. The correction are given for coordinates defined in an (arbitrarily chosen but now fixed) meta-frame coordinate system.

Currently, the best distortion map allows a recovery of the position with an rms of $<5$ mas.

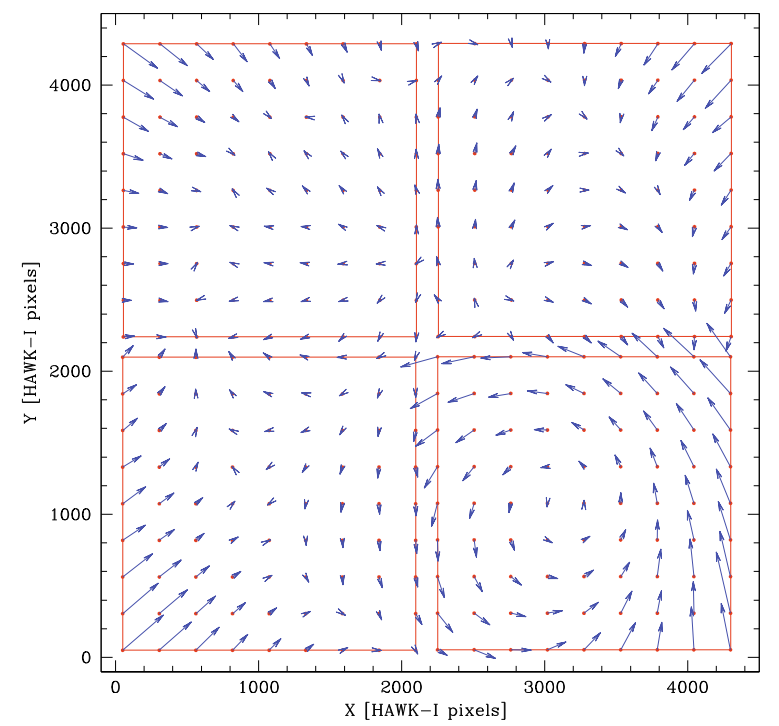

Fig. 12. HAWK-I distortion map. The red boxes show the positions of the single HAWK-I chips in the meta-chip system. The red dots are our grid points in the look-up table used to represent the geometrical distortion. The arrows give the sizes (exaggerated by a factor 100) and the directions of the correction to be applied to the grid-points to bring them into a distortion free frame. The correction for any pixels in the detector can be obtained with a bi-linear interpolation of the 4 closest grid-points.

\subsubsection{Image quality}

The image quality over the HAWK-I field is very homogeneous.

We evaluated the image quality on images taken in very good seeing conditions: down to an average image quality of $\sim 0.25^{\prime \prime}$ FWHM across the field, no trend in systematic elongation or preferred orientation of the PSF is detectable. Only for a combined image with an average image quality of $\sim 0.23^{\prime \prime}$ did we observe a $\sim 0.01^{\prime \prime}$ (i.e. $5 \%$ ) degradation of the image quality towards the edges of the $7.5^{\prime} \times 7.5^{\prime}$ field that could be attributed to the HAWK-I optics.

We conclude that for most observation, the user will not notice significant changes of the PSF across the entire field.

\subsection{The HAWK-I pipeline}

The reduction of HAWK-I data is common to near-infrared imagers and relatively straight forward. ESO provides a documented pipeline for the basic reduction steps (see Jung et al. 2008). The data reduction recipes performed by the pipeline are summarised with notes below.

- hawki_img_dark: the dark recipe sorts input data automatically by DIT and NDIT keywords and produces for each pair a master dark and bad (cold) pixel map.

- hawki_img_flat: the flat-field recipe sorts the input data by filter, and for uses for each a series of twilight flat-fields in order to build a illumination ramp for each pixel. The normalised slope of each pixel is stored in a master flat. Further, the recipe produces a bad (hot) pixel map, a statistics table and the fit error image for each master flat.

- hawki_img_zpoint: the recipe applies the flat-field and bad pixel map(s) to standard star data. It assumes that a standard star was placed in turn at the centre of each frame. Using the coordinates in the header, it identifies the observing 

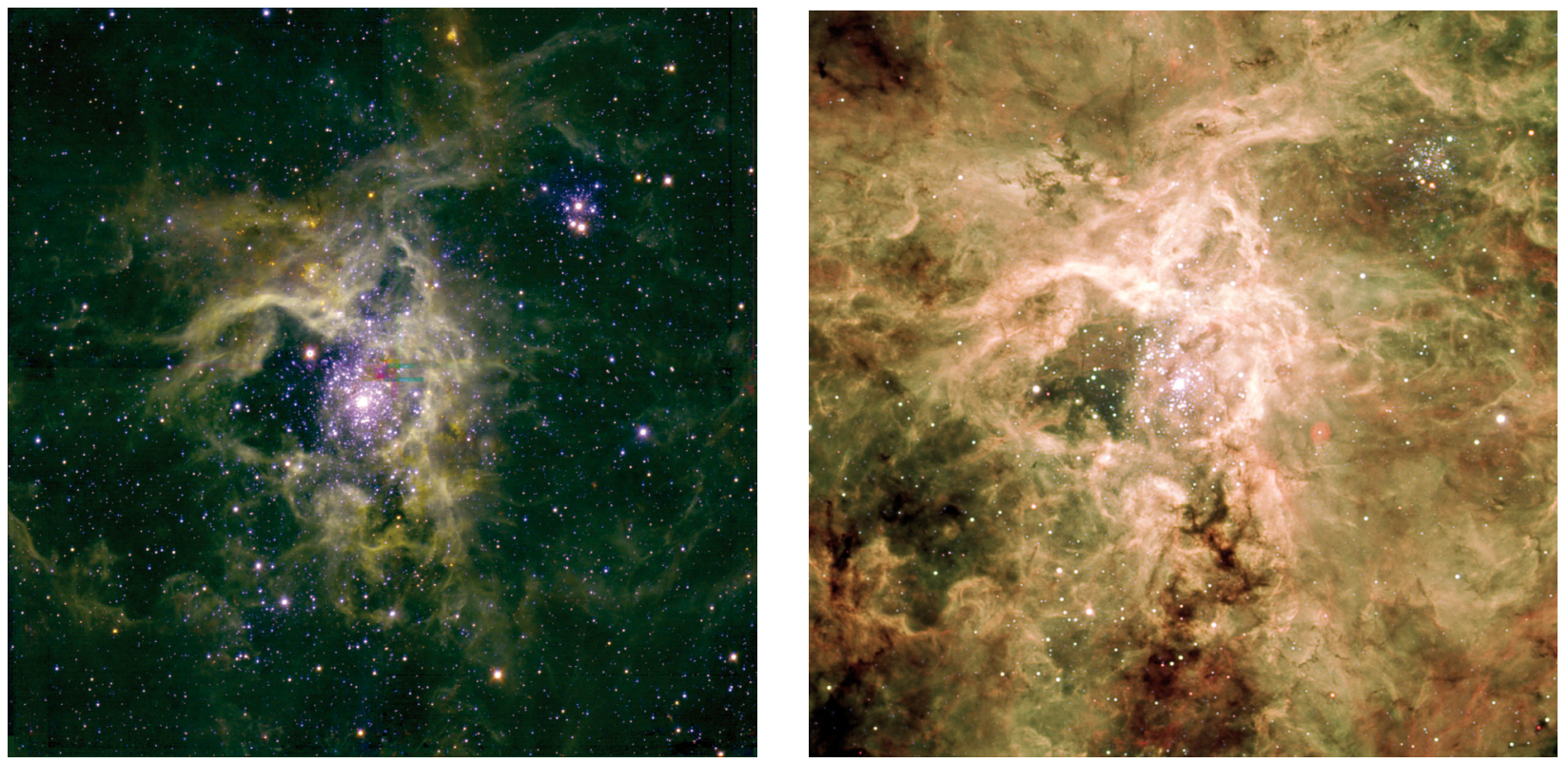

Fig. 13. A comparison of HAWK-I (left) and FORS (the work-horse optical imager of the VLT) (right) showing the Tarantula nebula.

sequence and the star in a list of standards, performs aperture photometry and tabulates the measured zeropoints.

- hawki_img_detlin: the recipe uses a series of Nasmyth screen flat-fields with increasing illumination in order to determine the detector linearity polynomial coefficients, as well as the error on the fit.

- hawki_img_illum: the input to this recipe is a series of standard star images in which the star was placed in a grid pattern in one or more quandrants. The images are reduced, as for the zero point recipe, aperture photometry is performed as a function of position on the chip and an illumination map of the detectors is computed.

- hawki_img_jitter: this recipe can take as input the result of any/all the previous ones and applies them science data resulting from the jitter and generic offset observations. The four quadrants are reduced separately, as well as combined into a single frame.

Besides the above recipes, the pipeline provides a number of utilities for usual tasks that users, reducing the data outside the pipeline, might want to apply. The following utilities are provided.

- hawki_util_distortion: the utility applies the distortion correction, using the distortion map discussed in Sect. 3.4.2.

- hawki_util_stitch: the utility combines/stiches the four quadrants to a single image.

- hawki_util_stdstars: this utility generates a standard stars catalogue (for the zero point and illumination recipes) from ascii files.

- hawki_util_gendist: this utility generates the distortion map used for the distortion correction.

A final result of the pipeline in shown in Fig. 13 as a composite of $Y, \mathrm{Br}_{\gamma}$, and $K_{\mathrm{s}}^{2}$.

\footnotetext{
${ }^{2}$ Note that the artifacts in the centre of the near-infrared image result from the dithering/observing strategy that was not optimally chosen at the time of the observations to produce a gap-less image.
}

\section{Outlook - ground-layer adaptive optics}

As mentioned above, HAWK-I has been design to keep open the possibility of a later use with an adaptive secondary mirror. The adaptive optic over a large field-of-view such as the one of HAWK-I will not give diffraction limited images. Instead a factor of two improvement of the seeing can reasonably been expected in the $K_{\mathrm{s}}$ band.

The secondary adaptive mirror of the VLT will operate with four lasers and one natural guide star. These lasers are mounted on the centre piece of the telescope and the laser direction will be rotated on the sky such that their images remain fixed with respect to the instrument field-of-view. In the case of HAWK-I, an optimal compromise between image quality and homogeneity of the PSF is achieved by positioning the four laser images at the centre of the four edges of the field-of-view.

The laser beams as well as the natural guide star beams will be picked-off in the ground-layer adaptive optics module (GRAAL, Arsenault et al. 2006) located between HAWK-I and the telescope adapter-rotator flange. The laser guide star wavefront sensors will have to compensate for the focus variations due to the finite altitude of the laser spots and the telescope position. The mechanism will also rotate the beam to match the sub-pupils between the wavefront sensors and the adaptive secondary mirror.

GRAAL with HAWK-I is expected to work routinely (starting around 2012) and to become the ground-layer adaptive optics facility for the VLT, improving even further HAWK-I's performances.

\section{Conclusions}

HAWK-I was successfully commissioned on the ESO VLT between August 2007 and February 2008. We believe that its wide field, excellent optical quality, extremely high throughput and cosmetically clean and high quantum efficiency detectors make it the most efficient near-infrared imager on an $8 \mathrm{~m}$ - to $10 \mathrm{~m}$-class telescope to date. Further enhancement in performance is expected in a few years when the telescope on which HAWK-I is mounted will be converted into an Adaptive Optics 
Facility providing ground layer correction over its complete field of $7.5^{\prime} \times 7.5^{\prime}$.

Following a highly productive phase of Science Verification which has demonstrated the high scientific quality of its images, HAWK-I was offered in the ESO call for proposals in September 2007 and has started regular science operation on April 1st, 2008.

Acknowledgements. We thank the referee for a careful reading and useful comments.

\section{References}

Arsenault, R., Hubin, N., Stroebele, S., et al. 2006, The Messenger, 123, 6
Anderson, J., Bedin, L. R., Piotto, G., Yadav, R. S., \& Bellini, A. 2006, A\&A, 454, 1029

Anderson, J., Bedin, L. R., et al., in preparation

Dalton, G. B., Caldwell, M., Ward, A. K., et al. 2006, SPIE, 6269, 30

Finger, G., Dorn, R. J., Eschbaumer, S., et al. 2008, SPIE, 7021, to be published Hawarden, T. G., Leggett, S. K., Letawsky, M. B., Ballantyne, D. R., \& Casali, M. M. 2001, MNRAS, 325, 563

Hewett, P. C., Warren, S. J., Leggett, S. K., \& Hodgkin, S. T. 2006, MNRAS, 367,454

Jung, Y., et al. 2008, The HAWK-I Pipeline User Manual (VLT-MAN-ESO19500-4407), ESO

Persson, S. E., Murphy, D. C., Krzeminski, W., Roth, M., \& Rieke, M. J. 1998, AJ, 116, 2475

Simons, D. A., \& Tokunaga, A. T. 2002, PASP, 114, 169

Tokunaga, A. T., \& Vacca, W. D. 2005, PASP, 117, 421 